\title{
Gamma-Ray Bursts at Neutrino Telescopes
}

\author{
Markus Ahlers* \\ Wisconsin IceCube Particle Astrophysics Center (WIPAC) and Department of Physics, \\ University of Wisconsin, Madison, WI 53706, USA \\ E-mail: mahlerseicecube.wisc.edu
}

Gamma-ray bursts (GRBs) are among the prime suspects as the sources of ultra-high energy (UHE) cosmic rays (CRs). It is believed that these sources are capable of accelerating nuclei to the extreme energies so far detected and are also able to supply enough power to sustain the energy density of UHE CRs. A smoking-gun signal of the acceleration of CRs in GRBs would be the emission of high-energy neutrinos from the decay of mesons that can be produced in photo-hadronic interactions in the presence of the burst or afterglow radiation. I will focus on the expected burst neutrino fluxes in the GRB fireball model. Recent results from the IceCube neutrino observatory on the emission of neutrinos from GRBs challenge the hypothesis that GRBs are the (main) sources of UHE CRs. I will summarize these results and their model implications.

Gamma-Ray Bursts 2012 Conference - GRB2012,

May 07-11, 2012,

Munich, Germany

${ }^{*}$ Speaker. 


\section{Introduction}

Many aspects of cosmic rays (CRs) remain a mystery even a century after their discovery. In particular the observation of ultra-high energy (UHE) CRs $(E>1 \mathrm{EeV})$ that extend up to energies of a few $100 \mathrm{EeV}$ are a challenge for astronomy and astrophysics. Unfortunately, the data on spectrum and composition of these UHE CRs is very limited and leaves room for many interpretations. Candidate sources of UHE CRs have to fulfill the necessary requirements of an efficient particle acceleration to these extreme energies [1] with an integrated power density of a few $10^{44} \mathrm{erg} \mathrm{Mpc}^{-3} \mathrm{yr}^{-1}$ above $10 \mathrm{EeV}$. Among the usual suspects are gamma-ray bursts (GRBs) occurring at a rate of a few hundreds per year in the visible Universe and releasing an energy of $\sim 10^{52}$ erg within seconds $[2,3,4]$.

The mere existence of UHE CRs is a strong motivation for neutrino astronomy at very high energies; it seems unavoidable that UHE CRs undergo hadronic interactions with radiation backgrounds and ambient matter prior to their arrival at Earth. Mesons produced in these interactions quickly decay and release a flux of high-energy neutrinos. The resulting neutrinos point back directly to the interaction site and are thus a smoking-gun signal for the CR accelerator. Moreover, they allow the study of very distant accelerators at energies that are not accessible by other messengers due to deflection and energy loss in magnetic fields (CRs/electrons) or absorption in radiation backgrounds (CRs/ $\gamma$-rays). However, the relation between the CR and neutrino luminosity of candidate sources depends on the particular source environment which is mostly model-dependent, i.e. either unknown or uncertain at best.

The flux of high-energy neutrinos at Earth is expected to be very faint and their interactions with matter are only very rare (the interaction length of multi- $\mathrm{TeV}$ neutrinos is of the order of the Earth's diameter). Neutrino observatories have thus to face the enormous challenge of observing and identifying very rare neutrino interactions in huge detection volumes. Secondary charged particles produced in weak interactions of neutrinos with nuclei can be identified by Cherenkov light emission in optically transparent media. This method has been successfully applied in Lake Baikal [5], the Mediterranean (ANTARES [6]) and the Antarctic glacier (AMANDA [7, 8], IceCube $[9,10])$. Coherent radio Cherenkov emission has been studied from the regolith of the Moon (GLUE [11]), in the Greenland ice sheet (FORTE [12]), and in Antarctic ice (ANITA [13], RICE [14]). Cosmic ray observatories can also identify neutrinos in CR air showers as deeply penetrating quasi-horizontal events and as electro-magnetic showers from Earth-skimming tau-neutrinos (HiRes [15] and Auger [16]).

Despite the large experimental effort, neutrino observatories have yet to identify the first extragalactic neutrino source and can so far only place upper limits on their fluxes. The strongest limits on the energy density of an isotropic spectrum of diffuse neutrinos are presently set in the $\mathrm{PeV}$ energy region by the IceCube observatory $[9,10]$. IceCube is a cubic-kilometer Cherenkov observatory located at the geographic South Pole. The main part of the detector is the "InIce" array of digital optical modules (DOM) looking for Cherenkov light emission from secondary particles produced in neutrino interactions. The DOMs are attached to supply and read-out cables - socalled "strings" - and deployed deep (below $1.5 \mathrm{~km}$ ) into the Antarctic ice. A set of 78 strings are placed at a distance of 125 meters on a triangular grid which results in its fiducial detector volume of roughly one cubic-kilometer. These strings have DOMs spaced every 17 meters, between 1450 




Figure 1: A sketch of the IceCube observatory. The main "InIce" observatory is supplemented by the low energy infill array "DeepCore". Hybrid observation of cosmic ray showers with "IceTop" allows for background rejection and cosmic ray studies. Also shown is the predecessor experiment "AMANDA".

and $2450 \mathrm{~m}$ in depth, giving a threshold for muon-neutrinos of about $100 \mathrm{GeV}$. Another 8 strings are placed near the center of IceCube and form "DeepCore" with a lower energy threshold of about $10 \mathrm{GeV}$ achieved by a decreased average DOM distance. In addition to the InIce array IceCube also possesses an air shower array called "IceTop" that consists of 80 pairs of water Cherenkov surface detectors. Hybrid observations of air showers in the InIce and IceTop arrays have mutual benefits, namely CR background rejection (for neutrino studies) and an improved air shower muon detection (for CR studies).

\section{Neutrino Emission in the GRB Fireball Scenario}

Gamma-ray bursts are one of the best-motivated candidate sources for high-energy cosmic ray and neutrino emission and Cherenkov observatories like IceCube are highly sensitive to the expected fluxes. Being cosmological sources it is likely that some GRBs appear in the neutrino observatories' field-of-view that are simultaneously detected by the Swift and/or Fermi satellite. In some cases follow-up observations allow for the determination of the source distance and other parameters needed for a precise estimate of the neutrino emission. Most importantly, the atmospheric background of the signal is significantly reduced if the predicted neutrino emission appears in coincidence with the short period of the burst.

We focus in the following on the fireball model that successfully accommodates the astronomical observations of many GRBs. The initial stage is set by the collapse of a compact massive star or a binary merger. Dissipation of gravitational energy leads to the creation of a hot fireball of electrons, photons and protons that is initially opaque to radiation. The hot plasma therefore expands by radiation pressure and particles are accelerated to a Lorentz factor $(\Gamma)$ that grows until 
the plasma becomes optically thin and produces the GRB display. From this point on the fireball is coasting with a Lorentz factor that is constant and depends on its baryonic load. The baryonic component carries the bulk of the fireball's kinetic energy. (See [17] for a recent review on theoretical models.).

The duration of the burst emission is set by the accretion time of matter onto the central engine. This accretion is likely in the form of a disk and the fireball expansion will be in the form of relativistic jets along the rotation axis of the progenitor system. The energetics and rapid time structure of the burst can be qualitativeley understood by shocks generated in the expanding fireball $[18,19,20]$. Here, the temporal variation of the $\gamma$-ray burst of the order of milliseconds can be interpreted as the collision of internal shocks with a varying baryonic load leading to differences in the bulk Lorentz factor. Electrons accelerated by first order Fermi acceleration radiate synchrotron $\gamma$-rays in the strong internal magnetic field and thus produce spikes in the burst spectra of the order of seconds [21,22]. The collision of the fireball with interstellar gas forms external shocks that can explain the GRB afterglow ranging from X-ray to the optical [23, 24].

High-energy neutrinos may be produced at various stages of the GRB emission. The collapse of a massive star is a likely progenitor of long-duration GRBs. Prior to the burst the fireball jet may burrow through the envelope of the stellar progenitor. Cosmic rays accelerated in substellar shocks can interact with matter or thermal photons to produce a flux of precursor neutrinos [25]. The neutrino flux depend on the particular progenitor type. Optimistic scenarios may produce high neutrino fluxes up to hundreds of $\mathrm{TeV}$. Interestingly, these neutrino emission will also be present for "failed" GRBs, i.e. core-collapse that do not produce a visible fireball emerging from the envelope. However, the estimated diffuse precursor flux from this "hidden" population is expected to be lower than the atmospheric neutrino background and will hence be hard to detect in diffuse neutrino searches [25]. This is different to the case of "successful" GRBs, where precursor neutrino can be looked for in the short window 10-100s prior to the burst with reduced atmospheric background. Neutrino emission associated with the afterglow has also been discussed by several authors [26, 27, 28]. If UHE CR acceleration happens in semi-relativistic reverse external shocks the neutrino spectrum from the interaction of CRs with the optical background can extend up to EeV energies depending on the maximal acceleration energy. Hence, if GRBs are the sources of UHE CRs this emission is particularly interesting for $\mathrm{EeV}$ neutrino astronomy like the proposed Askaryan Radio Array [29] (ARA) with highest sensitivity in the EeV energy range.

The most promising neutrino signal for the detection at IceCube are those neutrinos produced in coincidence with the short episode of $\gamma$-ray emission [30, 31, 32]. In the fireball model the burst is produced by synchrotron emission of electrons that are Fermi-accelerated in internal shocks. Protons that are also present in the fireball are co-accelerated and interact with the burst photons. The neutrino signal will follow the optical depth for $p \gamma$ interactions up to energies where synchrotron loss of mesons and muons prior to decay becomes relevant. For typical GRB parameters the neutrino signal will peak in the $100 \mathrm{TeV}$ to $10 \mathrm{PeV}$ energy region [30]. This is the region where IceCube is most sensitive and present neutrino limits are the strongest. We will discuss in the following in more detail how the expected burst neutrino signal relates to the internal GRB parameters. 


\section{Burst Neutrino Emission}

If protons are present in the fireball shock they should be co-accelerated with the electrons during the burst emission. Fermi-acceleration predict that the spectrum of accelerated protons in the fireball follows a power-law, $J_{\mathrm{CR}} \propto E^{-\gamma}$ with $\gamma \simeq 2$, up to a maximal energy which is typically set by the dynamical or synchrotron cooling time-scale. It is even possible (though not necessary in the following) to accelerate protons up to the energies required by the observed UHE CR spectrum.

The non-thermal CR spectrum can interact with the burst photons and produce a high-energy flux of pions that on decay give rise to the burst neutrino emission. The internal photon spectrum can be inferred from the observed luminosity $L_{\gamma}$ and relates to the internal energy density $U_{\gamma}^{\prime}$ as

$$
U_{\gamma}^{\prime} \simeq \frac{L_{\gamma}}{4 \pi} \frac{1}{r_{i}^{2} \Gamma^{2}} \simeq \int \mathrm{d} \varepsilon^{\prime} \varepsilon^{\prime} n_{\gamma}^{\prime}\left(\varepsilon^{\prime}\right)
$$

where $r_{i}$ is the internal shock radius that can be related to the observed variability time-scale $t_{v}$ and fireball Lorentz factor $\Gamma$, i.e. $r_{i} \simeq 2 \Gamma^{2} t_{v}$. Here and in the following, primed quantities refer to values in the co-moving plasma frame, whereas unprimed quantities are reserved for the observer's frame. The photon density in the co-moving and observer frame are related by $n_{\gamma}^{\prime}\left(\varepsilon^{\prime}\right)=n_{\gamma}\left(\Gamma \varepsilon^{\prime}\right)$. We will also express the fireball quantities in terms of "benchmark" values $L_{\gamma}=10^{52} L_{\gamma, 52} \mathrm{erg} / \mathrm{s}$, $\Gamma=10^{2.5} \Gamma_{2.5}$ and $t_{v}=0.01 t_{v,-2} \mathrm{~s}$.

We approximate in the following the $p \gamma$ cross section by interactions via the $\Delta$-resonance with mass $m_{\Delta} \simeq 1.232 \mathrm{GeV}$. The decay width $\Gamma_{\Delta} \simeq 0.11 \mathrm{GeV}$ is much smaller than the resonance mass and hence the narrow-width approximation can be applied. The optical depth is then approximately

$$
\tau_{p \gamma}\left(E_{p}^{\prime}\right)=\frac{t_{\mathrm{dyn}}^{\prime}}{t_{p \gamma}^{\prime}\left(E_{p}\right)} \simeq t_{\mathrm{dyn}}^{\prime}\left(\frac{\pi}{2} \frac{\Gamma_{\Delta} \sigma_{0} m_{\Delta}^{3}}{m_{\Delta}^{2}-m_{p}^{2}}\right) \frac{m_{p}^{2}}{E_{p}^{\prime 2}} \int_{\varepsilon_{\min }^{\prime}} \frac{\mathrm{d} \varepsilon^{\prime}}{\varepsilon^{\prime 2}} n_{\gamma}^{\prime}\left(\varepsilon^{\prime}\right),
$$

where $\varepsilon_{\min }^{\prime}=\left(m_{\Delta}^{2}-m_{p}^{2}\right) / 4 E_{p}^{\prime}$ and $\sigma_{0} \simeq 34 \mu \mathrm{b}$. The dynamical time-scale is given by the size of the shock, $t_{\mathrm{dyn}}^{\prime} \simeq r_{i} / 2 \Gamma$. Pion production hence follows

$$
E_{\pi}^{2} J_{\pi}\left(E_{\pi}\right) \simeq\left(1-e^{-\kappa \tau_{p \gamma}\left(E_{p} / \Gamma\right)}\right) E_{p}^{2} J_{p}\left(E_{p}\right) \equiv f_{\pi} E_{p}^{2} J_{p}\left(E_{p}\right) .
$$

Here, we introduce the inelasticity $\kappa \simeq 0.2$ with $E_{\pi}=\kappa E_{p}$ and the energy-dependent pion fraction $f_{\pi}$.

For small optical depths we can approximate $f_{\pi} \simeq \kappa \tau_{p \gamma}$. The minimal photon frequency $\varepsilon_{\min }^{\prime}$ for resonant interactions scales inversely with the proton energy $E_{p}^{\prime}$. Hence, if we approximate the burst emission via the Band spectrum with slopes $a \simeq-1$ and $b \simeq-2$ below and above the peak frequency, respectively, the optical depth (3.2) follows $\tau_{p \gamma} \propto\left(E_{p}^{\prime}\right)^{-b-1} \simeq E_{p}^{\prime}$ and $\tau_{p \gamma} \propto\left(E_{p}^{\prime}\right)^{-a-1} \simeq$ 1 at low and high proton energies, respectively. The proton break energy in the observatory frame is approximately $E_{p, b} \simeq 2 \Gamma_{2.5}^{2} / \varepsilon_{\mathrm{pk}, \mathrm{MeV}} \mathrm{PeV}$ where $\varepsilon_{\mathrm{pk}, \mathrm{MeV}}$ is the peak photon energy in $\mathrm{MeV}$.

The final burst neutrino spectrum are given by the decay of the charged pions as $\pi^{+} \rightarrow$ $\mu^{+} v_{\mu} \rightarrow e^{+} v_{e} \bar{v}_{\mu} v_{\mu}$. The neutrino break from the peak of the burst spectrum is $\kappa E_{p, b} / 4$ per neutrino flavor or

$$
E_{v, b} \simeq 100 \frac{\Gamma_{2.5}^{2}}{\varepsilon_{\mathrm{pk}, \mathrm{MeV}}} \mathrm{TeV}
$$


Synchrotron losses of pions and muons before their decay introduces a steepening of the neutrino spectrum $\propto E_{v}^{-2}$. The magnetic field can be estimated via the bolometric energy fractions $\varepsilon_{e}=$ $U_{e}^{\prime} / U_{\text {kin }}^{\prime}$ and $\varepsilon_{B}=U_{B}^{\prime} / U_{\text {kin }}^{\prime}$ in terms of the total kinetic energy of the fireball, i.e. $B^{2} / 2=U_{B}^{\prime}=$ $\left(\varepsilon_{B} / \varepsilon_{e}\right) U_{\gamma}^{\prime}$. The final (all flavor) neutrino spectrum is hence given by

$$
E_{v}^{2} J_{v}\left(E_{v}\right) \simeq \frac{1}{4} \frac{K}{1+K} f_{\pi} \sum_{v=v_{\mu}, \bar{v}_{\mu}, v_{e}} \frac{1}{1+\left(E_{v} / E_{v, \mathrm{~s}}\right)^{2}} E_{p}^{2} J_{p}\left(E_{p}\right),
$$

where $E_{v, \mathrm{~s}}$ is the critical neutrino energies above which synchrotron losses of the parent muon and pion before decay are relevant,

$$
E_{v, \mathrm{~s}}=\left(\frac{\varepsilon_{e,-1} \Gamma_{2.5}^{8} t_{v,-2}^{2}}{\varepsilon_{B,-1} L_{\gamma, 52}}\right)^{1 / 2} \times \begin{cases}20 \mathrm{PeV} & \left(v_{\mu}\right) \\ 1 \mathrm{PeV} & \left(\bar{v}_{\mu}, v_{e}\right) .\end{cases}
$$

The pre-factor in (3.5) accounts for the fact that each neutrino carries a quarter of the pions energy and $K /(1+K)$ accounts for the relative multiplicity of charged to neutral pions with $K=1 / 2$ for the $\Delta$-resonance. In summary, for a $\mathrm{CR}$ spectrum following $E^{-2}$ and a typical Band spectrum the neutrino production spectrum of each flavor in Eq. (3.5) follows $E^{-1}$ below the production break $E_{v, b}, E^{-4}$ above the synchrotron break $E_{v, \mathrm{~s}}$ and $E^{-2}$ in between.

For a known variability time-scale $t_{v}$, Lorentz-factor $\Gamma$, and equipartition parameters $\varepsilon_{e}$ and $\varepsilon_{B}$ we can calculate the neutrino emission of individual GRBs relative to the CR proton production. There are hence two possibilities for the absolute normalization of the spectra. (i) Introducing the proton equipartition parameter $\varepsilon_{p}=U_{p}^{\prime} / U_{\text {kin }}^{\prime}$ we can relate the proton spectrum to the observed luminosity of individual GRBs via the proton-to-electron energy ratio $\varepsilon_{p} / \varepsilon_{e}$. Note that this scenario does neither assume that GRBs are a dominant contributor of the observed spectrum of UHE CRs nor that it is actually capable of producing UHE CRs. (The maximal energy of accelerated protons does however enters the neutrino normalization via the relation of $J_{p}$ to the internal energy density $U_{p}^{\prime}$.) (ii) Alternatively, assuming that GRBs are the main sources of UHE CRs we can use the observed CR spectrum to predict the diffuse burst neutrino emission of GRBs. This method typically assumes average values of the internal shock parameters that are consistent with to acceleration of CRs in the GRBs to the observed energies of a few $100 \mathrm{EeV}$.

\section{Neutrino Diagnostics of the UHE CR Hypothesis}

The IceCube observatory has recently published the results of a search for neutrino emission in coincidence with GRBs in the period from April 2008 to May 2010 [34, 33] (see also Ref. [35] in these proceedings). The full detector was still under construction during this epoch and the data was taken with the 40 and 59 string sub-detectors available at that time. A total of 215 northern GRBs were included in the final analysis of IC-40/IC-59. Neutrino emission was studied via a "model-dependent" and "model-independent" analysis. The "model-independent" search of IC-59 included 85 additional GRBs in the southern sky.

The model-dependent analysis was tailored to look for burst neutrino emission from an individual burst with an emission spectrum following Eq. (3.5). The non-observation of neutrinos from this GRB sample translates into an upper bound on the possible stacked burst neutrino flux 

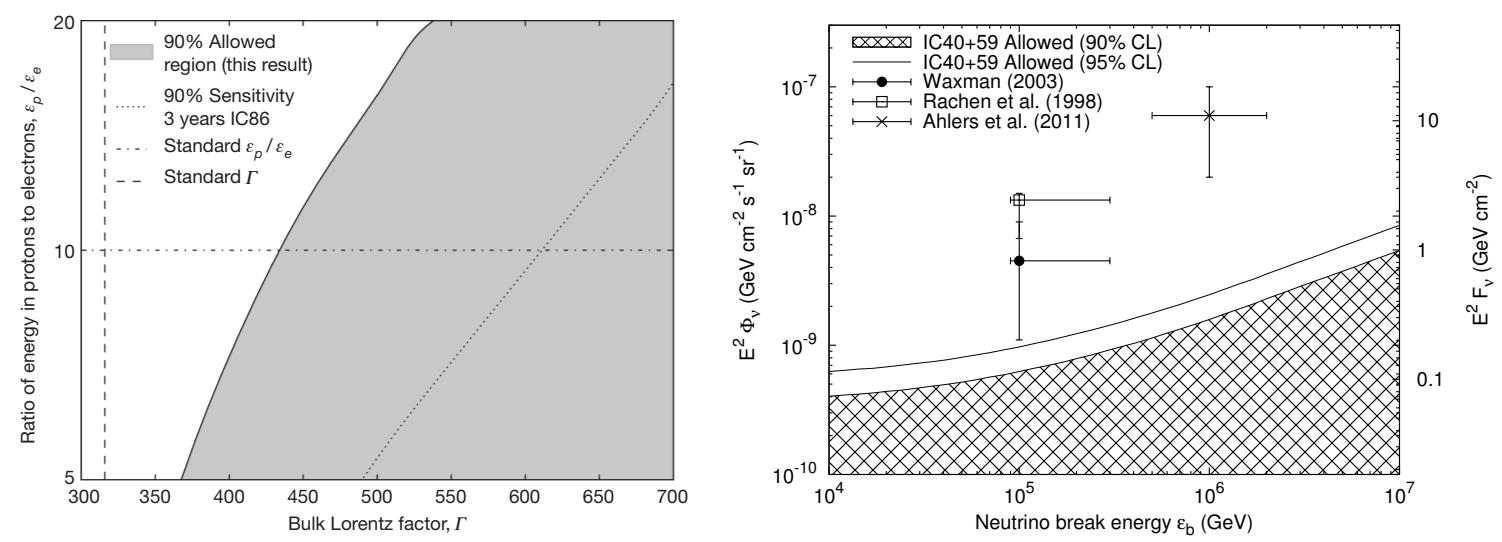

Figure 2: Figures from Ref. [33]. Left: Upper limits on GRB fireball parameters from the model-dependent analysis. Right: Upper limits on the normalization of a diffuse flux of prompt neutrinos derived from the model-independent result.

from the sum of all sources. The expected flux of neutrinos based on a normalization to the observed $\gamma$-ray luminosity as in Ref. [36] (model $(i)$ ) is a factor 3-4 higher than the upper flux limit, thus ruling out the model. Note, however, that this prediction is based on benchmark values of the otherwise unknown GRB parameters, like the Lorentz boost or the energy ratio of protons to electrons. The null result translates hence in a more general context to a limit on GRB model parameters. This is shown in the left plot of Fig. 2. Note, however, that in a serious of recent publications [37, 38, 39, 40] it was argued that the model predictions used in [34, 33] based on Ref. [36] should be revised to lower values accounting for bolometric correction factors (see also Ref. [41] in these proceedings). It is important to stress that the limits inferred in IceCube's model-dependent analysis are independent of these corrections, since these corrections don't effect the shape of the burst spectrum. However, the sensitivity of the model-dependent search to GRB model parameters becomes weaker with these corrections.

IceCube also performed a "model-independent" analysis which was looking for general neutrino events above background during a sliding time-window $|\Delta t|<10^{4} \mathrm{~s}$ relative to the burst. This analysis is also capable of detecting neutrino emission in the precursor and afterglow phases described earlier. The analysis found no excess of neutrino events above background and an upper limit was set on the stacked neutrino flux. This stacked point source limit can be rescaled to an upper limit on the diffuse burst neutrino emission of GRBs. The corresponding upper limit is shown in the right plot of Fig. 2 in terms of the first break of the neutrino spectrum (" $\varepsilon_{b}$ " in the plot), which depend on the Lorentz factor as $\varepsilon_{b} \propto \Gamma^{2}$. The rescaling to a diffuse flux assumes 667 bursts per year. Also indicated in the plot are predictions of the diffuse neutrino emission based on a normalization via the UHE CR spectrum [30, 42, 43] (model (ii)). All these models are excluded by the limit.

The IceCube results are hence challenging models of UHE CR production in GRBs. Note that the model predictions shown in Fig. 2 are realizations of the same fireball phenomenology for specific benchmark values. As indicated in the plots, the sensitivity of IceCube decreases with an increasing Lorentz factor since the observed spectrum shifts to higher energies $\left(E_{v} \propto \Gamma^{2}\right)$. 
It is hence not possible at the present stage to fully exclude the hypothesis that GRBs are the sources of UHE CR protons. Benchmark models, however, have to be revised in light of the recent IceCube results. Models that relate the neutrino and CR spectrum via neutron emission [42, 43] are independent of the pion fraction and are largely ruled out.

\section{Acknowledgements}

I would like to thank the organizers of GRB 2012 for a stimulating conference and their support.

\section{References}

[1] A. Hillas, The Origin of Ultrahigh-Energy Cosmic Rays, Ann.Rev.Astron.Astrophys. 22 (1984) 425-444.

[2] A. Levinson and D. Eichler, Baryon Purity in Cosmological Gamma-Ray Bursts as a Manifestation of Event Horizons, Astrophys.J. 418 (Nov., 1993) 386.

[3] M. Vietri, On the acceleration of ultrahigh-energy cosmic rays in gamma-ray bursts, Astrophys.J. 453 (1995) 883-889, [astro-ph/9506081].

[4] E. Waxman, Cosmological origin for cosmic rays above $10^{19} \mathrm{eV}$, Astrophys.J. 452 (1995) L1-L4, [astro-ph/9508037].

[5] BAIKAL Collaboration, V. Aynutdinov et al., Search for a diffuse flux of high-energy extraterrestrial neutrinos with the nt200 neutrino telescope, Astropart.Phys. 25 (2006) 140-150, [astro-ph/0508675].

[6] Antares Collaboration, J. A. Aguilar et al., Search for a diffuse flux of high-energy $v_{\mu}$ with the ANTARES neutrino telescope, Phys.Lett. B696 (2011) 16, [arXiv:1011.3772].

[7] IceCube Collaboration, A. Achterberg et al., Multi-year search for a diffuse flux of muon neutrinos with AMANDA-II, Phys.Rev. D76 (2007) 042008, [arXiv: 0705.1315$].$

[8] IceCube Collaboration, M. Ackermann et al., Search for Ultra High-Energy Neutrinos with AMANDA-II, Astrophys.J. 675 (2008) 1014-1024, [arXiv: 0711 . 3022].

[9] IceCube Collaboration, R. Abbasi et al., Constraints on the Extremely-high Energy Cosmic Neutrino Flux with the IceCube 2008-2009 Data, Phys.Rev. D83 (2011) 092003, [arXiv:1103.4250].

[10] IceCube Collaboration, R. Abbasi et al., A Search for a Diffuse Flux of Astrophysical Muon Neutrinos with the IceCube 40-String Detector, Phys.Rev. D84 (2011) 082001, [arXiv: 1104.5187 ].

[11] P. W. Gorham, C. Hebert, K. Liewer, C. Naudet, D. Saltzberg, et al., Experimental limit on the cosmic diffuse ultrahigh-energy neutrino flux, Phys.Rev.Lett. 93 (2004) 041101, [astro-ph/ 0310232 ].

[12] N. G. Lehtinen, P. W. Gorham, A. R. Jacobson, and R. A. Roussel-Dupre, FORTE satellite constraints on ultra-high energy cosmic particle fluxes, Phys.Rev. D69 (2004) 013008, [astro-ph/ 0309656 ].

[13] ANITA Collaboration, P. Gorham et al., Observational Constraints on the Ultra-high Energy Cosmic Neutrino Flux from the Second Flight of the ANITA Experiment, Phys.Rev. D85 (2012) 049901, [arXiv:1011.5004].

[14] I. Kravchenko, C. Cooley, S. Hussain, D. Seckel, P. Wahrlich, et al., Rice limits on the diffuse ultrahigh energy neutrino flux, Phys.Rev. D73 (2006) 082002, [ast ro-ph / 0601148 ]. 
[15] HiRes Collaboration, K. Martens, HiRes Estimates and Limits for Neutrino Fluxes at the Highest Energies, arXiv:0707.4417.

[16] Pierre Auger Collaboration, J. Abraham et al., Limit on the diffuse flux of ultra-high energy tau neutrinos with the surface detector of the Pierre Auger Observatory, Phys.Rev. D79 (2009) 102001, [arXiv:0903.3385].

[17] P. Meszaros, Gamma-Ray Bursts, Rept.Prog.Phys. 69 (2006) 2259-2322, [ast ro-ph/ 0605208 ].

[18] A. Shemi and T. Piran, The appearance of cosmic fireballs, Astrophys.J. 365 (1990) L55-L58.

[19] M. Rees and P. Meszaros, Relativistic fireballs - energy conversion and time - scales, Mon.Not.Roy.Astron.Soc. 258 (1992) 41-43.

[20] P. Meszaros and M. Rees, Relativistic fireballs and their impact on external matter - Models for cosmological gamma-ray bursts, Astrophys.J. 405 (1993) 278.

[21] M. Rees and P. Meszaros, Unsteady outflow models for cosmological gamma-ray bursts, Astrophys.J. 430 (1994) L93-L96, [astro-ph/ 9404038 ].

[22] B. Paczynski and G. Xu, Neutrino bursts from gamma-ray bursts, Astrophys.J. 427 (1994) 708-713.

[23] P. Meszaros, M. Rees, and H. Papathanassiou, Spectral properties of blast wave models of gamma-ray burst sources, Astrophys.J. 432 (1994) 181-193, [astro-ph/9311071].

[24] P. Meszaros and M. Rees, Delayed GeV emission from cosmological gamma-ray bursts: Impact of a relativistic wind on external matter, Mon.Not.Roy.Astron.Soc. 269 (1994) L41, [astro-ph/9404056].

[25] S. Razzaque, P. Meszaros, and E. Waxman, High energy neutrinos from gamma-ray bursts with precursor supernovae, Phys.Rev.Lett. 90 (2003) 241103, [astro-ph / 0212536 ].

[26] K. Murase and S. Nagataki, High Energy Neutrino Flash from Far-UV/X-ray Flares of Gamma-Ray Bursts, Phys.Rev.Lett. 97 (2006) 051101, [astro-ph / 0604437 ].

[27] K. Murase, High energy neutrino early afterglows gamma-ray bursts revisited, Phys.Rev. D76 (2007) 123001, [arXiv:0707.1140].

[28] S. Razzaque, O. Mena, and C. D. Dermer, Prompt TeV emission from cosmic rays accelerated by gamma-ray bursts interacting with a surrounding stellar wind, Astrophys.J. 691 (2009) L37-L40.

[29] ARA Collaboration, P. Allison, J. Auffenberg, R. Bard, J. Beatty, D. Besson, et al., Design and Initial Performance of the Askaryan Radio Array Prototype EeV Neutrino Detector at the South Pole, Astropart.Phys. 35 (2012) 457-477, [arXiv:1105.2854].

[30] E. Waxman and J. N. Bahcall, High-energy neutrinos from cosmological gamma-ray burst fireballs, Phys.Rev.Lett. 78 (1997) 2292-2295, [astro-ph/9701231].

[31] K. Murase and S. Nagataki, High energy neutrino emission and neutrino background from gamma-ray bursts in the internal shock model, Phys.Rev. D73 (2006) 063002, [astro-ph / 0512275 ].

[32] L. A. Anchordoqui, D. Hooper, S. Sarkar, and A. M. Taylor, High-energy neutrinos from astrophysical accelerators of cosmic ray nuclei, Astropart.Phys. 29 (2008) 1-13, [astro-ph/ 0703001 ].

[33] IceCube Collaboration, R. Abbasi et al., An absence of neutrinos associated with cosmic-ray acceleration in $\gamma$-ray bursts, Nature 484 (2012) 351-353, [arXiv : 1204 . 4219].

[34] IceCube Collaboration, R. Abbasi et al., Limits on Neutrino Emission from Gamma-Ray Bursts with the 40 String IceCube Detector, Phys.Rev.Lett. 106 (2011) 141101, [arXiv: 1101.1448 ]. 
[35] A. Homeier, Recent icecube results from searches for transient neutrino sources, Proceedings of GRB 2012, Munich, Germany (2012).

[36] D. Guetta, M. Spada, and E. Waxman, On the neutrino flux from gamma-ray bursts, Astrophys.J. 559 (2001) 101, [astro-ph/0102487].

[37] Z. Li, Note on the Normalization of Predicted GRB Neutrino Flux, Phys.Rev. D85 (2012) 027301, [arXiv:1112.2240].

[38] S. Hummer, P. Baerwald, and W. Winter, Neutrino Emission from Gamma-Ray Burst Fireballs, Revised, arXiv:1112.1076.

[39] P. Baerwald, S. Hummer, and W. Winter, Systematics in the Interpretation of Aggregated Neutrino Flux Limits and Flavor Ratios from Gamma-Ray Bursts, Astropart.Phys. 35 (2012) 508-529, [arXiv:1107.5583].

[40] H.-N. He, R.-Y. Liu, X.-Y. Wang, S. Nagataki, K. Murase, et al., Icecube non-detection of GRBs: Constraints on the fireball properties, arXiv:1204.0857.

[41] P. Baerwald, Neutrinos from grbs, and the connection to gamma-ray observations, Proceedings of GRB 2012, Munich, Germany (2012).

[42] J. P. Rachen and P. Meszaros, Cosmic rays and neutrinos from gamma-ray bursts, AIP Conf.Proc. 428 (1997) 776, [astro-ph/9811266].

[43] M. Ahlers, M. Gonzalez-Garcia, and F. Halzen, GRBs on probation: testing the UHE CR paradigm with IceCube, Astropart.Phys. 35 (2011) 87-94, [arXiv: 1103.3421 ]. 